\title{
Current-induced reversal of anomalous Hall conductance in twisted bilayer graphene
}

\author{
Ying $\mathrm{Su}^{1}$ and Shi-Zeng $\operatorname{Lin}^{1}$ \\ ${ }^{1}$ Theoretical Division, T-4 and CNLS, Los Alamos National Laboratory, Los Alamos, New Mexico 87545, USA
}

(Dated: November 25, 2020)

\begin{abstract}
It is observed experimentally that the sign of the Hall resistance can be flipped by a dc electric current in the twisted bilayer graphene (TBG) at 3/4 filling of the four-fold degenerate conduction flat bands. The experiment implies a switching of the valley polarization (VP) and topology in TBG. Here we present a theory on the current-induced switching of VP and topology. The presence of current in the bulk causes the redistribution of electron occupation in bands near the Fermi energy, which then deforms and shifts the band dispersion due to the Coulomb interaction. Above a critical current, the original occupied and empty bands can be swapped resulting in the switching of VP and topology.
\end{abstract}

Introduction.-The electronic band structure is modified significantly compared to that in a single layer graphene, when two layers of graphene are stacked together [1]. The bandwidth can be controlled by the misalignment angle between two layers [2,3]. At certain twisted angles called magic angles, the energy bands near the Fermi surface are extremely flat [3], where the Coulomb interaction becomes dominant over the kinetic energy of electrons. It is expected that novel quantum states enabled by the strong electronic correlation will emerge. Indeed, the correlated insulating state and superconductivity have been observed experimentally in the magicangle twisted bilayer graphene (TBG) $[4,5]$. Furthermore, the insulating state can have nontrivial topology which is manifested by the intrinsic quantum anomalous Hall effect (QAHE) [6, 7]. The twisted multilayer graphene therefore becomes an important platform to explore the physics of strong electronic correlation and topology [4-77].

The QAHE with orbital ferromagnetism was observed at $3 / 4$ filling of the upper flat bands in TBG recently $[6,7]$. In the absence of electronic interaction, the electronic bands of TBG have the four-fold degeneracy associated with the valley and spin degrees of freedom. The strong electronic interaction in flat bands lifts the valley and spin degeneracy, that results in the valley-spin-polarized insulating state responsible for the QAHE at 3/4 filling [20, 58, 70-73]. The nontrivial topology of the insulating state is characterized by the Chern number $C= \pm 1$, where the sign of Chern number depends on which valley is polarized. Remarkably, as demonstrated in experiments, the sign of Hall conductance can be flipped by a dc current, that indicates the switching of Chern number and valley polarization (VP) [6, 7]. Several theoretical pictures are proposed to explain the experiments [7, 49, 78].

In this paper, we propose a mechanism of switching of VP and Chern number in TBG by electric currents in the bulk. The current causes the redistribution of the electron occupation in otherwise fully occupied or empty bands, which deforms and shifts the band dispersion with respect to the Fermi energy $E_{F}$ due to the Coulomb interaction. Above a threshold current, the major part of the originally empty (occupied) band when current is absent is pushed below (above) $E_{F}$. The bands then are swapped after relaxation when the current is removed, that hence results in flipping of the VP and topol- ogy.

Toy model.-To facilitate the understanding of switching of VP by electric currents, we use a simple toy model to demonstrate the physical picture. Here we consider a twoband model described by the Hamiltonian

$$
\mathcal{H}=\sum_{k, \tau}\left(\varepsilon_{k, \tau}-\lambda \partial_{k} \varepsilon_{k, \tau}\right) c_{k, \tau}^{\dagger} c_{k, \tau}+V \sum_{k_{1}, k_{2}} c_{k_{1},+}^{\dagger} c_{k_{1},+} c_{k_{2},-}^{\dagger} c_{k_{2},-}
$$

where $\tau= \pm$ is the effective valley index labeling the two energy bands and $V$ is the repulsive intervalley interaction. The model has time-reversal symmetry (TRS), $\varepsilon_{k, \tau}=\varepsilon_{-k,-\tau}$. The current in the system can be introduced by the Lagrangian multiplier $\lambda$, which can be derived by considering a system with a threading magnetic flux [79]. It can also be understood intuitively in terms of the semi-classical Boltzmann transport theory [79]. The self-consistent mean-field Hamiltonian is $\mathcal{H}_{M F}=\sum_{k, \tau} E_{k, \tau} c_{k, \tau}^{\dagger} c_{k, \tau}$ with $E_{k, \tau}=\varepsilon_{k, \tau}-\lambda \partial_{k} \varepsilon_{k, \tau}+\Delta_{-\tau}$ and $\Delta_{\tau}=V \sum_{k} f\left(E_{k, \tau}\right)$, where $f\left(E_{k, \tau}\right)$ is the Fermi distribution function.

Consider the schematic band dispersions shown in Fig. 1(a). The band with valley + is above that with valley - as a consequence of the spontaneous symmetry breaking due to the intervalley interaction. The system is an insulator at half filling. To introduce current, a fraction of electrons in the lower band must be pumped into the upper band. Because the electron self-energy depends on the electron occupation, the part of - band with group velocity opposite to the current direction rises while the + band shifts downwards. The majority of the + band can be below the - band above a critical current, as shown in Fig. 1(b). After removing the current, the system then relaxes into a state with $+(-)$ band occupied (empty), and thus the VP is switched, as shown in Fig. 1(c).

To describe the phase transition associated with the switching of VP, we introduce the VP order parameter $\delta=\left(\Delta_{+}-\right.$ $\left.\Delta_{-}\right) / 2$. Close to the transition temperature $T_{c}$ when $\delta$ is small, the Ginzburg-Landau free energy of the system can be expanded as [79]

$$
\mathcal{F}=\mathcal{F}_{0}+\alpha_{1} \delta+\alpha_{2} \delta^{2}+\alpha_{3} \delta^{3}+\alpha_{4} \delta^{4} .
$$

Because both current and $\delta$ are odd under time reversal, it requires $\alpha_{1,3}(\lambda)=-\alpha_{1,3}(-\lambda)$ and $\alpha_{2,3}(\lambda)=\alpha_{2,4}(-\lambda)$. Under the 

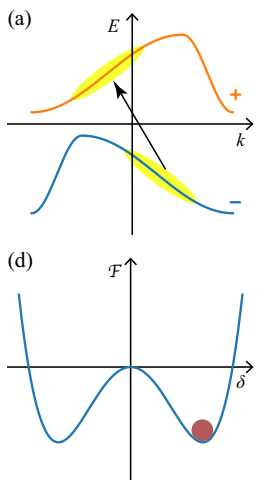

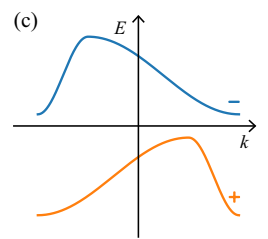

(e)

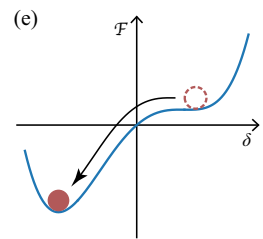

(b)

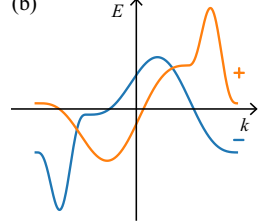

(f)

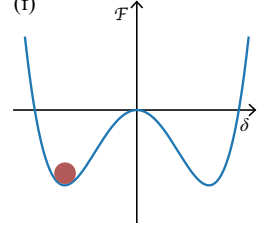

FIG. 1. (a)-(c) Schematic band structures for $\lambda=0, \lambda \geq \lambda_{c}$, and $\lambda=0$, respectively. (d)-(f) Free energy profiles correspond to (a)-(c). The two energy bands are labeled by the effective valley index \pm . (a) and (c) represent the two degenerate ground states with opposite VP. (b) is obtained from (a) by introducing the current, and (c) is obtained from (b) by removing the current. The current induced pumping of electrons from the lower band to the upper band is indicated by the arrow and is highlighted in (a). The switching of VP and vanishing of energy barrier in the presence of current is exhibited in (e).

inversion operation within each valley $\hat{\Gamma}_{v}$, i.e. $\varepsilon_{k, \tau} \rightarrow \varepsilon_{-k, \tau}, \delta$ is even while the current is odd. If the system has the symmetry associated with $\hat{\Gamma}_{v}$, then $\alpha_{1,3}(\lambda)=\alpha_{1,3}(-\lambda)$, which immediately implies $\alpha_{1,3}(\lambda)=0$. Therefore this intravalley inversion symmetry must be broken, i.e. $\varepsilon_{k, \tau} \neq \varepsilon_{-k, \tau}$, in order to switch the valley polarization by current.

It is clear from Eq. (2) that the system has two degenerate ground states with opposite VP, which are separated by an energy barrier, as shown in Figs. 1(d) and 1(f). The presence of current lifts the degeneracy by increasing the energy of one valley-polarized state and reducing the energy of the other one, as shown in Fig. 1(e). Above a critical current, the energy barrier vanishes. The VP of the system is switched if it is initiated at a state, that becomes unstable under the current [see Fig. 1(e)]. On the other hand, if the system sits in the other state that remains the energy minimal under the current, there is no switching of VP. Thus the switching of VP depends on the direction of current.

TBG.-We then apply our physical picture to TBG with a small twist angle. In TBG, no symmetry guarantees $\varepsilon_{\boldsymbol{k}, \tau}=$ $\varepsilon_{-\boldsymbol{k}, \tau}$ and therefore it is possible to switch the VP by electric currents. We employ the continuum model Hamiltonian [3]

$$
\mathcal{H}_{\boldsymbol{k}, \tau}=\left(\begin{array}{cc}
h_{1, \tau}(\boldsymbol{k}) & T_{\tau}(\boldsymbol{r}) \\
T_{\tau}^{\dagger}(\boldsymbol{r}) & h_{2, \tau}(\boldsymbol{k})
\end{array}\right),
$$

where $\tau= \pm$ is the valley index and $h_{l, \tau}(\boldsymbol{k})$ is the low-energy effective Dirac Hamiltonian of the $l$-th layer graphene. Under the twist that the first (second) layer is rotated by $\theta / 2(-\theta / 2)$, the Dirac Hamiltonian becomes

$$
h_{l, \tau}(\boldsymbol{k})=\mathcal{R}( \pm \tau \theta / 2) \hbar v_{F}\left(\boldsymbol{k}-\tau \boldsymbol{K}_{l}\right) \cdot \boldsymbol{\sigma}_{\tau} \mathcal{R}( \pm \tau \theta / 2)^{-1},
$$

where $\mathcal{R}(\theta)=e^{-i \theta \sigma_{z} / 2}$ is the rotation operator and $\sigma_{\tau}=$ $\left(\tau \sigma_{x}, \sigma_{y}\right)$ is the Pauli matrix for sublattice degree of freedom.

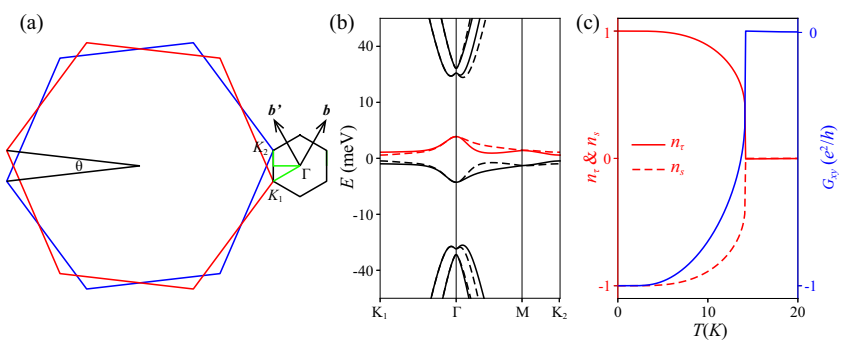

FIG. 2. (a) Schematic BZs of the TBG (black hexagon) and of two graphene layers (red and blue hexagons). (b) Low-energy band structure of the TBG along the high symmetry path. The solid and dashed energy bands are from the $\pm \mathrm{K}$ valleys, respectively. The upper flat bands are marked by red color. (c) $n_{\tau}$ (red solid line), $n_{s}$ (red dashed line), and Hall conductance $G_{x y}$ (blue solid line) are plotted as a function of the temperature $T$.

$\boldsymbol{K}_{l}$ are the corners of Moire Brouillon zone (BZ) as shown in Fig. 2(a). The interlayer coupling is described by

$$
\begin{aligned}
& T_{\tau}(\boldsymbol{r})=T_{\tau}^{(0)}+e^{-i \tau \boldsymbol{b} \cdot \boldsymbol{r}} T_{\tau}^{(1)}+e^{-i \tau \boldsymbol{b}^{\prime} \cdot \boldsymbol{r}} T_{\tau}^{(2)}, \\
& T_{\tau}^{(n)}=w_{A A} \sigma_{0}+w_{A B} \cos \left(\frac{2 \pi n}{3}\right) \sigma_{x}+\tau w_{A B} \sin \left(\frac{2 \pi n}{3}\right) \sigma_{y},
\end{aligned}
$$

where $\boldsymbol{b}\left(\boldsymbol{b}^{\prime}\right)$ are the primitive reciprocal lattice vectors of the TBG [see Fig. 2(a)]. Due to the lattice relaxation, the interlayer tunneling strength is different for the $\mathrm{AA}$ and $\mathrm{AB}$ stacking regions, where $w_{A A}=79.7 \mathrm{meV}$ and $w_{A B}=97.5$ $\mathrm{meV}$ [13]. The Fermi velocity is $\hbar v_{F} / a=2.1354 \mathrm{eV}$ where $a=0.246 \mathrm{~nm}$ is the lattice constant of single layer graphene. $\mathcal{H}_{k, \pm}$ of the $\pm K$ valleys are related by TRS. In the experiments, the TBG with $\theta=1.15^{\circ}$ is aligned with a hBN substrate $[6,7]$ that induces a sublattice potential onto the first layer graphene as $h_{1, \tau} \rightarrow h_{1, \tau}+\Delta_{A B} \sigma_{z} / 2$ through the interlayer coupling. We choose the sublattice potential $\Delta_{A B}=20 \mathrm{meV}$ in this study. The Hamiltonian Eq. (3) is expressed in the real space representation with $\boldsymbol{k}=-i \nabla_{\boldsymbol{r}}$ being the momentum operator. Under the plane wave expansion and truncation of reciprocal space to the fourth shell, the low-energy bands of the TBG are well converged and shown in Fig. 2(b), where the energy bands from the $\pm K$ valley are represented by solid and dashed lines, respectively. There are eight flat bands including the degenerate spin degree of freedom around charge neutrality. The lattice relaxation isolates the flat bands from remote bands and the substrate-induced sublattice potential gaps the upper flat bands from the lower flat bands by breaking the $C_{2} \mathcal{T}$ symmetry that enables the nonzero valley Chern number [12]. Here $C_{2}$ and $\mathcal{T}$ denote the two-fold rotation and time-reversal symmetries, respectively.

Here we focus on the $3 / 4$ filling of the upper flat bands [the red bands in Fig. 2(b)], where the QAHE emerges [6, 7]. By projecting the Coulomb interaction onto the upper flat bands, the Hamiltonian of TBG becomes

$$
\mathcal{H}_{0}=\sum_{\boldsymbol{k}, \tau, s}\left(\varepsilon_{\boldsymbol{k}, \tau}-\mu\right) c_{\boldsymbol{k}, \tau, s}^{\dagger} c_{\boldsymbol{k}, \tau, s}+\frac{1}{2 A} \sum_{\boldsymbol{q}} \rho(\boldsymbol{q}) V(\boldsymbol{q}) \rho(-\boldsymbol{q}),
$$


where $\varepsilon_{k, \tau}$ denotes the spin-degenerate bare dispersion for the upper flat bands given by $\mathcal{H}_{k, \tau}\left|\psi_{k, \tau}\right\rangle=\varepsilon_{k, \tau}\left|\psi_{k, \tau}\right\rangle, \mu$ is the chemical potential, and $A$ is the area of the system. $s=\uparrow$ or $\downarrow$ represents the spin degree of freedom. $\rho(\boldsymbol{q})=$ $\sum_{k, \boldsymbol{k}^{\prime}, \tau, s}\left\langle\psi_{\boldsymbol{k}, \tau}\left|e^{i \boldsymbol{q} \cdot \boldsymbol{r}}\right| \psi_{\boldsymbol{k}^{\prime}, \tau}\right\rangle c_{\boldsymbol{k}, \tau, s}^{\dagger} c_{\boldsymbol{k}^{\prime}, \tau, s}$ is the density operator, and $V(\boldsymbol{q})=e^{2} \tanh (|\boldsymbol{q}| d) / 2 \epsilon|\boldsymbol{q}|$ is the screened Coulomb potential, where $\epsilon$ is the dielectric constant and $d$ is the distance between TBG and metallic gates. Here we take $d=40 \mathrm{~nm}$ following the experiment [7].

With the self-consistent Hartree-Fock approximation, the dispersion of the upper flat bands are corrected by the Coulomb interaction as

$$
\begin{aligned}
& E_{\boldsymbol{k}, \tau, s}=\varepsilon_{\boldsymbol{k}, \tau}-\mu \\
& +\frac{1}{A} \sum_{\boldsymbol{q}, \boldsymbol{k}^{\prime}, \tau^{\prime}, s^{\prime}}\left\langle\psi_{\boldsymbol{k}, \tau}\left|e^{i \boldsymbol{q} \cdot \boldsymbol{r}}\right| \psi_{\boldsymbol{k}, \tau}\right\rangle V(\boldsymbol{q})\left\langle\psi_{\boldsymbol{k}^{\prime}, \tau^{\prime}}\left|e^{-i \boldsymbol{q} \cdot \boldsymbol{r}}\right| \psi_{\boldsymbol{k}^{\prime}, \tau^{\prime}}\right\rangle f\left(E_{\boldsymbol{k}^{\prime}, \tau^{\prime}, s^{\prime}}\right) \\
& -\frac{1}{A} \sum_{\boldsymbol{q}, \boldsymbol{k}^{\prime}}\left\langle\psi_{\boldsymbol{k}, \tau}\left|e^{i \boldsymbol{q} \cdot \boldsymbol{r}}\right| \psi_{\boldsymbol{k}^{\prime}, \tau}\right\rangle V(\boldsymbol{q})\left\langle\psi_{\boldsymbol{k}^{\prime}, \tau}\left|e^{-i \boldsymbol{q} \cdot \boldsymbol{r}}\right| \psi_{\boldsymbol{k}, \tau}\right\rangle f\left(E_{\boldsymbol{k}^{\prime}, \tau, s}\right)
\end{aligned}
$$

By solving the self-consistent equation, we get four degenerate valley-spin-polarized ground states at $3 / 4$ filling. In the ground states, one of the four flat bands is above the other three that results in an insulating state when $\mu$ is tuned to be inside the gap. The experimentally measured energy gap is $\Delta / k_{B} \approx 27 K$ [7], that can be reproduced by our approach with $\epsilon=58.6 \epsilon_{0}$, where $\epsilon_{0}$ is the vacuum permittivity. The energy gap depends on the gate distance $d$. As $d$ decreases, the screening of the Coulomb interaction is enhanced and the energy gap is reduced [79].

We define $n_{\tau}=\sum_{s}\left(n_{+, s}-n_{-, s}\right)$ and $n_{s}=\sum_{\tau}\left(n_{\tau, \uparrow}-n_{\tau, \downarrow}\right)$ to characterize the valley and spin polarization, respectively. $n_{\tau, s}$ is the occupation number of the flat band with the valleyspin indices $(\tau, s)$. We consider a symmetry-breaking state with $n_{\tau}=-n_{s}=1$ at zero temperature, as shown in Fig. 3 . The four flat bands have nonzero Chern numbers $C= \pm 1$, which are opposite for $\pm K$ valleys as a consequence of TRS. Therefore, the VP at 3/4 filling ensures the QAHE, which is manifested by the quantized Hall conductance $G_{x y}= \pm e^{2} / h$ as observed in experiments [7]. Because the Hamiltonian Eq. (6) has the spin SU(2) symmetry and valley $U(1)$ symmetry, the long-range spin order is destroyed by thermal fluctuations according to the Mermin-Wagner theorem, while the long-range valley order is allowed [80]. As temperature increases, the mean field $n_{\tau}, n_{s}$, and $G_{x y}$ vanish together above a critical temperature $T_{c}=14.1 \mathrm{~K}$, as shown in Fig. 2(c). Above $T_{c}$, the system becomes a simple metal.

We then study the effect of current and magnetic field on the electron dispersion. The current is introduced through the Lagrange multiplier as before and the Hamiltonian becomes

$$
\mathcal{H}=\mathcal{H}_{0}-\sum_{k, \tau, s}\left(\lambda_{s} \partial_{k_{\alpha}} \varepsilon_{k, \tau}+g \mu_{B} s_{z} B+M_{\boldsymbol{k}, \tau} B\right) c_{k, \tau, s}^{\dagger} c_{k, \tau, s},
$$

where $\lambda_{s}$ denotes the Lagrange multiplier for the current with spin $s$ and along the $\alpha$ direction, $s_{z}= \pm 1 / 2$ is the spin quantum
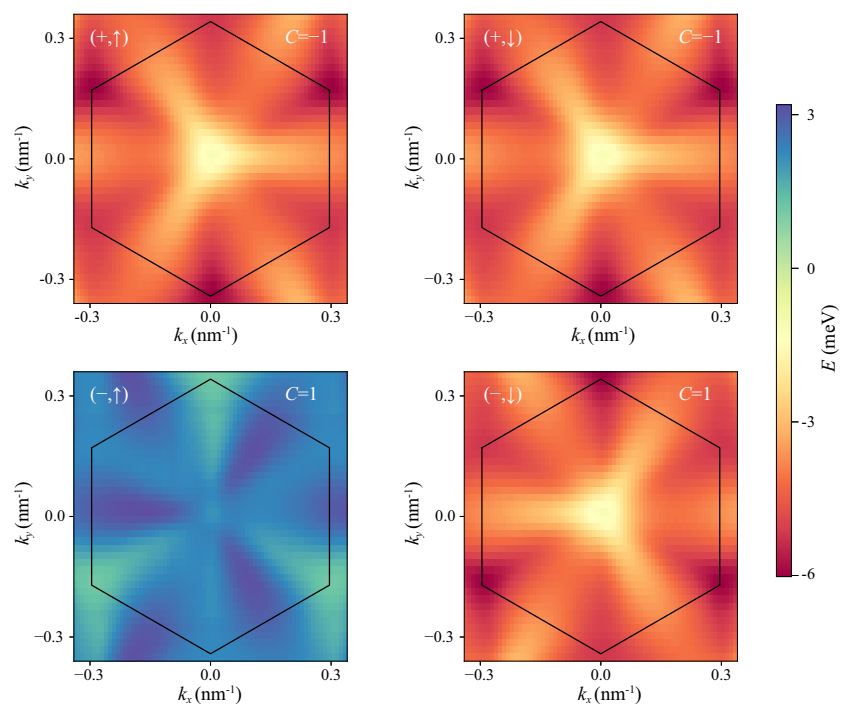

FIG. 3. The four upper fat bands labeled by the valley-spin in$\operatorname{dex}(\tau, s)$ are shown for the insulating valley-spin-polarized state obtained from the Hatree-Fock approximation. The Chern numbers of the flat bands are displayed. The black hexagon encloses the moiré BZ.

number, and $B$ represents the perpendicular magnetic field. $M_{\boldsymbol{k}, \tau}$ is the $\boldsymbol{k}$-resolved orbital magnetization

$$
M_{\boldsymbol{k}, \tau}=i \frac{e}{2 \hbar}\left\langle\nabla_{\boldsymbol{k}} \psi_{\boldsymbol{k}, \tau}\left|\times\left(2 \mu_{0}-\varepsilon_{\boldsymbol{k}, \tau}-\mathcal{H}_{\boldsymbol{k}, \tau}\right)\right| \nabla_{\boldsymbol{k}} \psi_{\boldsymbol{k}, \tau}\right\rangle \cdot \hat{\boldsymbol{e}}_{z},
$$

that is generated by the self rotation of Bloch states and includes also the boundary contribution [81-84]. Here $\mu_{0}$ is the chemical potential corresponding to the $3 / 4$ filling of the bare dispersion, and $\hat{\boldsymbol{e}}_{z}$ is a unit vector perpendicular to TBG. The orbital magnetic moment is spin-degenerate and valleycontrasting. Thus the symmetry breaking state at $3 / 4$ filling has the polarized orbital magnetic moment, and exhibits orbital ferromegnetism. To be concrete, we focus on the current along the $x$ direction and take the valley-spin-polarized ground state in Fig. 3 as the initial state. Because the flat bands with spin down are fully filled, the current can only be conducted by the spin up bands. Hence we fix $\lambda_{\downarrow}=0$ in the following. In this case, the Hatree-Fock approximation yields the self-consistent equation in the same form as Eq. (7) but with the replacement $\varepsilon_{\boldsymbol{k}, \tau} \rightarrow \varepsilon_{\boldsymbol{k}, \tau}-\lambda_{s} \partial_{k_{x}} \varepsilon_{\boldsymbol{k}, \tau}-g \mu_{B} s_{z} B-M_{\boldsymbol{k}, \tau} B$.

We first focus on the effect of current on VP and Hall conductance in the absence of magnetic field $B=0$. The analysis in Eq. (2) reveals that the switching of VP by currents is of the first order phase transition, and therefore hysteresis is expected. We sweep the current by changing $\lambda$ continuously and solve the corresponding self-consistent equations. The current density through the TBG is $J=\frac{e}{A h} \sum_{\boldsymbol{k}, \tau, s} \frac{\partial \varepsilon_{\boldsymbol{k}, \tau}}{\hbar \partial k_{x}} f\left(E_{\boldsymbol{k}, \tau, s}\right)$, where $h=0.6 \mathrm{~nm}$ is the thickness of TBG. In Fig. 4(a), we show that $J$ increases monontonically with $\lambda$. The results of $n_{\tau}, n_{s}$, and $G_{x y}$ at $T=13 \mathrm{~K}$ as a function of $J$ are displayed in Figs. 4(b) and 4(c), where the hysteretic behavior of VP and Hall 

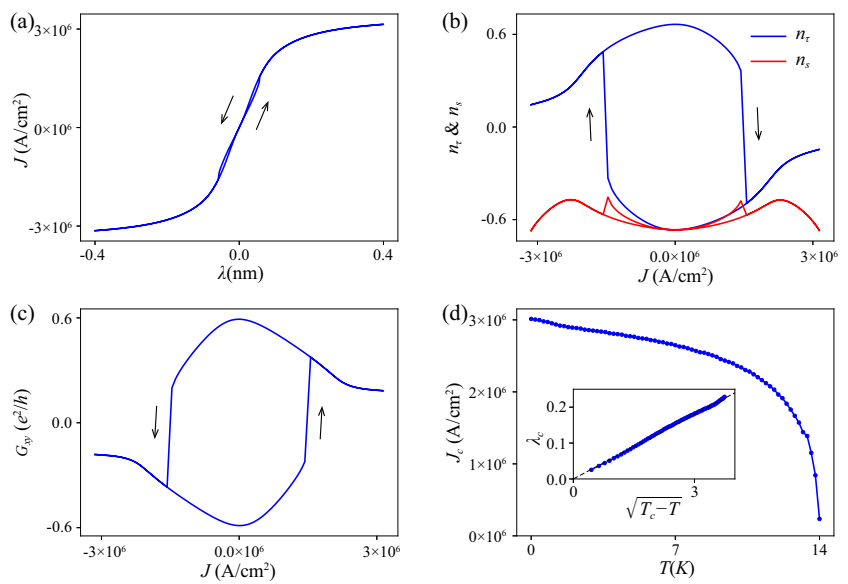

FIG. 4. (a) Current density $J$ as a function of $\lambda$ at $T=13 K$. (b) and (c) The corresponding order parameters $n_{\tau, s}$ and Hall conductance $G_{x y}$ as a function of $J$. (d) The dependence of critical current density $J_{c}$ on $T$. The inset shows $\lambda_{c}$ vs $\sqrt{T_{c}-T}$.

conductance appears as expected. Start with the initial state in Fig. 3 with $n_{\tau} \approx 0.6$ and $G_{x y} \approx-0.6 e^{2} / h$ (here $n_{\tau}$ and $G_{x y}$ are not saturated because of the nonzero $T$.), and then increase $J$, both $n_{\tau}$ and $G_{x y}$ change sign through a sharp jump at the critical current density $J_{c}$ (and the corresponding $\lambda_{c}$ ). If we remove the current (by setting $\lambda=0$ ) after the jump, the system then relaxes into an insulating state with opposite $n_{\tau}$ and $G_{x y}$. There is no switching of spin polarization because the bands with down spin remain fully occupied. Therefore, our results demonstrate that the VP and topology can be switched by a current larger than $J_{c}$. The results for $T=0$ are presented in Ref. [79].

Here $\lambda_{c}$ and $J_{c}$ are determined by the energy barrier separating different valley-polarized states. As $T$ increases, the energy barrier decreases, and hence $\lambda_{c}$ and $J_{c}$ decreases with

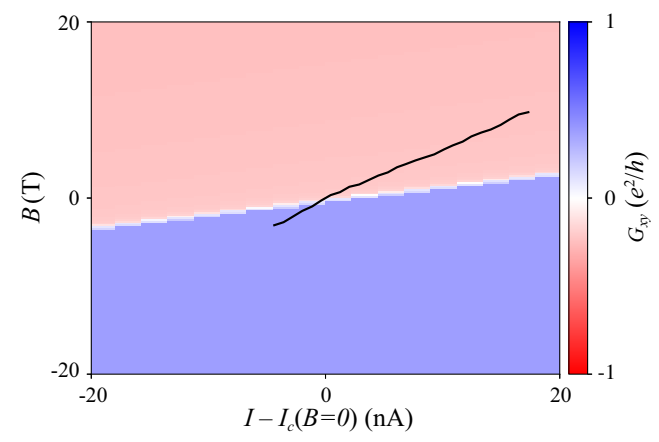

FIG. 5. Density plot of the Hall conductance $G_{x y}$ as a function of current $I$ and magnetic field $B$. The positive and negative $G_{x y}$ are for opposite valley polarization. The black line is the phase boundary extracted from Ref. [7] that separates different valley-polarized states. Here we offset $I$ by the critical current $I_{c}$ at $B=0$ to make a direct comparison of the phase boundaries.
$T$. The dependence of $\lambda_{c}$ and $J_{c}$ on $T$ are shown in Fig. 4(d). As can be derived from Eq. (2), we have the following scaling relations $\mathcal{F} \sim\left(T_{c}-T\right)^{2}, \delta \sim \sqrt{T_{c}-T}$, and $\alpha_{3} \sim \lambda$. Therefore $\lambda_{c} \sim \sqrt{T_{c}-T}$ consistent with the numerical results shown in the inset of Fig. 4(d).

The energy barrier is affected by the magnetic field through the Zeeman coupling with spin and orbital magnetic moments in Eq. (8). In TBG, the orbital magnetic moment is dominant and is valley-contrasting. The energy gap of the insulating valley-spin-polarized state scales linearly with $B$. To study the dependence of critical current $I_{c}$ on $B$, we show $G_{x y}$ as a function of current $I$ for different $B$ at $T=13 \mathrm{~K}$ in Fig. 5 . The switching of VP and topology at $I_{c}$ is featured by the jump of $G_{x y}$ where $G_{x y}$ changes sign. $I_{c}$ as a function of $B$ indicated by the black line in Fig. 5 is extracted from Ref. [7]. Both the experimental result and our numerical simulation show a linear dependence of $I_{c}$ on $B$.

Discussion and summary.- - Here we compare our theory to the other proposals [7, 49, 78]. In Ref. [7], the authors attributed the switching of VP due to the chiral edge current, where the asymmetric edges tilt the free energy difference for two otherwise degenerate VP states. However, the switching of the VP requires overcoming the bulk energy barrier. For a large system size with a single domain, the edge contribution is not enough to overcome the bulk energy barrier [79]. One may argue that the switching involves multiple domains. In this picture, the domain walls must percolate the whole system in order to conduct current. In Ref. [49], He et al. argued that strain due to the substrate can break the $C_{3}$ symmetry down to $C_{1}$ symmetry. The magnetoelectric effect then can allow to change the magnetization by electric field, and switch the VP. This requires an electric field throughout the system and finite quasiparticle lifetime, and therefore does not apply directly to the QAHE states. In Ref. [78], the authors considered domain walls between different VP. They argued that the electric current can couple to the domain wall described by the valley pseudospin and drive the domain wall into motion. The motion of domain wall results in the expansion of domains with one VP at the expense of the domains with the opposite VP, and eventually flips the VP.

In our approach, we highlight the effect of the bulk current. A bulk current in the QAHE state is possible either due to thermally activated quasiparticle or when the electric field exceeds a threshold value determined by the gap [79]. Our scenario can be tested experimentally by studying devices with a single domain. The single domain state can be prepared by polarizing the devices with external magnetic field at low temperature [7]. One then measures the longitudinal currentvoltage curve and the corresponding Hall conductance, and compares to the theoretical prediction in Fig. 4.

Our theory can be generalized to the multiple domains case. The mechanism proposed here offers a way to change the energy for different VP domains by current, which facilitates the nucleation of the energetically favored VP in the presence of fluctuations. Therefore, the threshold switching current identified for a single domain when the energy 
barrier vanishes completely corresponds to the superheating/supercooling field.

In summary, we propose a mechanism of the switching of VP and topology in TBG by bulk electric currents. The current causes the redistribution of electron occupation, that lifts the degeneracy and even overcomes the energy barrier between different valley-spin-polarized states through the Coulomb interaction. Our theory can be generalized to other strongly correlated two dimensional materials with valley polarization.

Acknowledgements.-The authors thank Fengcheng Wu and Di Xiao for helpful discussion. Computer resources for numerical calculations were supported by the Institutional Computing Program at LANL. This work was carried out under the auspices of the U.S. DOE NNSA under contract No. 89233218CNA000001 through the LDRD Program, and was supported by the Center for Nonlinear Studies at LANL and Institute for Materials Science (IMS) at LANL through IMS Rapid Response. S. Z. L. was also supported by the U.S. Department of Energy, Office of Science, Basic Energy Sciences, Materials Sciences and Engineering Division, Condensed Matter Theory Program.

[1] A. H. Castro Neto, F. Guinea, N. M. R. Peres, K. S. Novoselov, and A. K. Geim, "The electronic properties of graphene," Rev. Mod. Phys. 81, 109-162 (2009).

[2] J. M. B. Lopes dos Santos, N. M. R. Peres, and A. H. Castro Neto, "Graphene bilayer with a twist: Electronic structure," Phys. Rev. Lett. 99, 256802 (2007).

[3] Rafi Bistritzer and Allan H. MacDonald, "Moiré bands in twisted double-layer graphene," PNAS 108, 12233-12237 (2011).

[4] Yuan Cao, Valla Fatemi, Ahmet Demir, Shiang Fang, Spencer L. Tomarken, Jason Y. Luo, Javier D. SanchezYamagishi, Kenji Watanabe, Takashi Taniguchi, Efthimios Kaxiras, Ray C. Ashoori, and Pablo Jarillo-Herrero, "Correlated insulator behaviour at half-filling in magic-angle graphene superlattices," Nature 556, 80-84 (2018).

[5] Yuan Cao, Valla Fatemi, Shiang Fang, Kenji Watanabe, Takashi Taniguchi, Efthimios Kaxiras, and Pablo Jarillo-Herrero, "Unconventional superconductivity in magic-angle graphene superlattices," Nature 556, 43-50 (2018).

[6] Aaron L. Sharpe, Eli J. Fox, Arthur W. Barnard, Joe Finney, Kenji Watanabe, Takashi Taniguchi, M. A. Kastner, and David Goldhaber-Gordon, "Emergent ferromagnetism near three-quarters filling in twisted bilayer graphene," Science $\mathbf{3 6 5}$, 605-608 (2019).

[7] M. Serlin, C. L. Tschirhart, H. Polshyn, Y. Zhang, J. Zhu, K. Watanabe, T. Taniguchi, L. Balents, and A. F. Young, "Intrinsic quantized anomalous hall effect in a moiré heterostructure," Science (2019), 10.1126/science.aay5533.

[8] Xiaobo Lu, Petr Stepanov, Wei Yang, Ming Xie, Mohammed Ali Aamir, Ipsita Das, Carles Urgell, Kenji Watanabe, Takashi Taniguchi, Guangyu Zhang, Adrian Bachtold, Allan H. MacDonald, and Dmitri K. Efetov, "Superconductors, orbital magnets and correlated states in magic-angle bilayer graphene," Nature 574, 653-657 (2019).
[9] Kyounghwan Kim, Ashley DaSilva, Shengqiang Huang, Babak Fallahazad, Stefano Larentis, Takashi Taniguchi, Kenji Watanabe, Brian J. LeRoy, Allan H. MacDonald, and Emanuel Tutuc, "Tunable moiré bands and strong correlations in small-twistangle bilayer graphene," PNAS 114, 3364-3369 (2017).

[10] Shengqiang Huang, Kyounghwan Kim, Dmitry K. Efimkin, Timothy Lovorn, Takashi Taniguchi, Kenji Watanabe, Allan H. MacDonald, Emanuel Tutuc, and Brian J. LeRoy, "Topologically protected helical states in minimally twisted bilayer graphene,” Phys. Rev. Lett. 121, 037702 (2018).

[11] Cenke Xu and Leon Balents, "Topological superconductivity in twisted multilayer graphene," Phys. Rev. Lett. 121, 087001 (2018).

[12] Hoi Chun Po, Liujun Zou, Ashvin Vishwanath, and T. Senthil, "Origin of mott insulating behavior and superconductivity in twisted bilayer graphene,” Phys. Rev. X 8, 031089 (2018).

[13] Mikito Koshino, Noah F. Q. Yuan, Takashi Koretsune, Masayuki Ochi, Kazuhiko Kuroki, and Liang Fu, "Maximally localized wannier orbitals and the extended hubbard model for twisted bilayer graphene," Phys. Rev. X 8, 031087 (2018).

[14] Jian Kang and Oskar Vafek, "Symmetry, maximally localized wannier states, and a low-energy model for twisted bilayer graphene narrow bands," Phys. Rev. X 8, 031088 (2018).

[15] Cheng-Cheng Liu, Li-Da Zhang, Wei-Qiang Chen, and Fan Yang, "Chiral spin density wave and $d+i d$ superconductivity in the magic-angle-twisted bilayer graphene," Phys. Rev. Lett. 121, 217001 (2018).

[16] J. F. Dodaro, S. A. Kivelson, Y. Schattner, X. Q. Sun, and C. Wang, "Phases of a phenomenological model of twisted bilayer graphene,” Phys. Rev. B 98, 075154 (2018).

[17] Hiroki Isobe, Noah F. Q. Yuan, and Liang Fu, "Unconventional superconductivity and density waves in twisted bilayer graphene,” Phys. Rev. X 8, 041041 (2018).

[18] Louk Rademaker and Paula Mellado, "Charge-transfer insulation in twisted bilayer graphene,” Phys. Rev. B 98, 235158 (2018).

[19] Francisco Guinea and Niels R Walet, "Electrostatic effects, band distortions, and superconductivity in twisted graphene bilayers," Proceedings of the National Academy of Sciences 115, 13174-13179 (2018).

[20] Ming Xie and A. H. MacDonald, "Nature of the correlated insulator states in twisted bilayer graphene," Phys. Rev. Lett. 124, 097601 (2020).

[21] Matthew Yankowitz, Shaowen Chen, Hryhoriy Polshyn, Yuxuan Zhang, K. Watanabe, T. Taniguchi, David Graf, Andrea F. Young, and Cory R. Dean, "Tuning superconductivity in twisted bilayer graphene," Science 363, 1059-1064 (2019).

[22] Alexander Kerelsky, Leo J McGilly, Dante M Kennes, Lede Xian, Matthew Yankowitz, Shaowen Chen, K Watanabe, $\mathrm{T}$ Taniguchi, James Hone, Cory Dean, et al., "Maximized electron interactions at the magic angle in twisted bilayer graphene," Nature 572, 95-100 (2019).

[23] Yuan Cao, Debanjan Chowdhury, Daniel Rodan-Legrain, Oriol Rubies-Bigorda, Kenji Watanabe, Takashi Taniguchi, T. Senthil, and Pablo Jarillo-Herrero, "Strange metal in magicangle graphene with near planckian dissipation," Phys. Rev. Lett. 124, 076801 (2020).

[24] Hryhoriy Polshyn, Matthew Yankowitz, Shaowen Chen, Yuxuan Zhang, K Watanabe, T Taniguchi, Cory R Dean, and Andrea F Young, "Large linear-in-temperature resistivity in twisted bilayer graphene," Nature Physics 15, 1011-1016 (2019).

[25] Guorui Chen, Aaron L. Sharpe, Eli J. Fox, Ya-Hui Zhang, Shaoxin Wang, Lili Jiang, Bosai Lyu, Hongyuan Li, Kenji 
Watanabe, Takashi Taniguchi, Zhiwen Shi, T. Senthil, David Goldhaber-Gordon, Yuanbo Zhang, and Feng Wang, "Tunable correlated chern insulator and ferromagnetism in a moiré superlattice," Nature 579, 56-61 (2020).

[26] Emilio Codecido, Qiyue Wang, Ryan Koester, Shi Che, Haidong Tian, Rui Lv, Son Tran, Kenji Watanabe, Takashi Taniguchi, Fan Zhang, Marc Bockrath, and Chun Ning Lau, "Correlated insulating and superconducting states in twisted bilayer graphene below the magic angle," Science Advances 5 (2019), 10.1126/sciadv.aaw9770, https://advances.sciencemag.org/content/5/9/eaaw9770.full.pdf.

[27] Yonglong Xie, Biao Lian, Berthold Jäck, Xiaomeng Liu, Cheng-Li Chiu, Kenji Watanabe, Takashi Taniguchi, B Andrei Bernevig, and Ali Yazdani, "Spectroscopic signatures of manybody correlations in magic-angle twisted bilayer graphene," Nature 572, 101-105 (2019).

[28] Yuhang Jiang, Xinyuan Lai, Kenji Watanabe, Takashi Taniguchi, Kristjan Haule, Jinhai Mao, and Eva Y Andrei, "Charge order and broken rotational symmetry in magic-angle twisted bilayer graphene," Nature 573, 91-95 (2019).

[29] Youngjoon Choi, Jeannette Kemmer, Yang Peng, Alex Thomson, Harpreet Arora, Robert Polski, Yiran Zhang, Hechen Ren, Jason Alicea, Gil Refael, et al., "Electronic correlations in twisted bilayer graphene near the magic angle," Nature Physics 15, 1174-1180 (2019).

[30] Xiaobo Lu, Petr Stepanov, Wei Yang, Ming Xie, Mohammed Ali Aamir, Ipsita Das, Carles Urgell, Kenji Watanabe, Takashi Taniguchi, Guangyu Zhang, et al., "Superconductors, orbital magnets and correlated states in magic-angle bilayer graphene," Nature 574, 653-657 (2019).

[31] S. L. Tomarken, Y. Cao, A. Demir, K. Watanabe, T. Taniguchi, P. Jarillo-Herrero, and R. C. Ashoori, "Electronic compressibility of magic-angle graphene superlattices," Phys. Rev. Lett. 123, 046601 (2019).

[32] Cheng Shen, Yanbang Chu, QuanSheng Wu, Na Li, Shuopei Wang, Yanchong Zhao, Jian Tang, Jieying Liu, Jinpeng Tian, Kenji Watanabe, Takashi Taniguchi, Rong Yang, Zi Yang Meng, Dongxia Shi, Oleg V. Yazyev, and Guangyu Zhang, "Correlated states in twisted double bilayer graphene," Nature Physics 16, 520-525 (2020).

[33] Xiaomeng Liu, Zeyu Hao, Eslam Khalaf, Jong Yeon Lee, Yuval Ronen, Hyobin Yoo, Danial Haei Najafabadi, Kenji Watanabe, Takashi Taniguchi, Ashvin Vishwanath, and Philip Kim, "Tunable spin-polarized correlated states in twisted double bilayer graphene," Nature 583, 221-225 (2020).

[34] Yuan Cao, Daniel Rodan-Legrain, Oriol Rubies-Bigorda, Jeong Min Park, Kenji Watanabe, Takashi Taniguchi, and Pablo Jarillo-Herrero, "Tunable correlated states and spin-polarized phases in twisted bilayer-bilayer graphene," Nature 583, 215220 (2020).

[35] Yi-Zhuang You and Ashvin Vishwanath, "Superconductivity from valley fluctuations and approximate so (4) symmetry in a weak coupling theory of twisted bilayer graphene," npj Quantum Materials 4, 1-12 (2019).

[36] Qing-Kun Tang, Lin Yang, Da Wang, Fu-Chun Zhang, and Qiang-Hua Wang, "Spin-triplet $f$-wave pairing in twisted bilayer graphene near $\frac{1}{4}$-filling,” Phys. Rev. B 99, 094521 (2019).

[37] J. González and T. Stauber, "Kohn-luttinger superconductivity in twisted bilayer graphene," Phys. Rev. Lett. 122, 026801 (2019).

[38] Ying Su and Shi-Zeng Lin, "Pairing symmetry and spontaneous vortex-antivortex lattice in superconducting twistedbilayer graphene: Bogoliubov-de gennes approach," Phys. Rev. B 98, 195101 (2018).
[39] Aline Ramires and Jose L. Lado, "Electrically tunable gauge fields in tiny-angle twisted bilayer graphene," Phys. Rev. Lett. 121, 146801 (2018).

[40] Grigory Tarnopolsky, Alex Jura Kruchkov, and Ashvin Vishwanath, "Origin of magic angles in twisted bilayer graphene," Phys. Rev. Lett. 122, 106405 (2019).

[41] Junyeong Ahn, Sungjoon Park, and Bohm-Jung Yang, "Failure of nielsen-ninomiya theorem and fragile topology in twodimensional systems with space-time inversion symmetry: Application to twisted bilayer graphene at magic angle," Phys. Rev. X 9, 021013 (2019).

[42] Zhida Song, Zhijun Wang, Wujun Shi, Gang Li, Chen Fang, and B. Andrei Bernevig, "All magic angles in twisted bilayer graphene are topological,” Phys. Rev. Lett. 123, 036401 (2019).

[43] Kasra Hejazi, Chunxiao Liu, Hassan Shapourian, Xiao Chen, and Leon Balents, "Multiple topological transitions in twisted bilayer graphene near the first magic angle," Phys. Rev. B 99, 035111 (2019).

[44] Yury Sherkunov and Joseph J. Betouras, "Electronic phases in twisted bilayer graphene at magic angles as a result of van hove singularities and interactions," Phys. Rev. B 98, 205151 (2018).

[45] Jian Kang and Oskar Vafek, "Strong coupling phases of partially filled twisted bilayer graphene narrow bands," Phys. Rev. Lett. 122, 246401 (2019).

[46] Kangjun Seo, Valeri N. Kotov, and Bruno Uchoa, "Ferromagnetic mott state in twisted graphene bilayers at the magic angle," Phys. Rev. Lett. 122, 246402 (2019).

[47] Yu-Ping Lin and Rahul M. Nandkishore, "Chiral twist on the high- $T_{c}$ phase diagram in moiré heterostructures," Phys. Rev. B 100, 085136 (2019).

[48] Teemu J. Peltonen, Risto Ojajärvi, and Tero T. Heikkilä, "Mean-field theory for superconductivity in twisted bilayer graphene,” Phys. Rev. B 98, 220504 (2018).

[49] Wen-Yu He, David Goldhaber-Gordon, and Kam Tuen Law, "Giant orbital magnetoelectric effect and current-induced magnetization switching in twisted bilayer graphene," Nature communications 11, 1650 (2020).

[50] Biao Lian, Zhijun Wang, and B. Andrei Bernevig, "Twisted bilayer graphene: A phonon-driven superconductor," Phys. Rev. Lett. 122, 257002 (2019).

[51] Young Woo Choi and Hyoung Joon Choi, "Strong electronphonon coupling, electron-hole asymmetry, and nonadiabaticity in magic-angle twisted bilayer graphene," Phys. Rev. B 98, 241412 (2018).

[52] Fengcheng Wu, A. H. MacDonald, and Ivar Martin, "Theory of phonon-mediated superconductivity in twisted bilayer graphene,” Phys. Rev. Lett. 121, 257001 (2018).

[53] Shubhayu Chatterjee, Nick Bultinck, and Michael P. Zaletel, "Symmetry breaking and skyrmionic transport in twisted bilayer graphene," Phys. Rev. B 101, 165141 (2020).

[54] Fengcheng Wu, Euyheon Hwang, and Sankar Das Sarma, "Phonon-induced giant linear-in- $t$ resistivity in magic angle twisted bilayer graphene: Ordinary strangeness and exotic superconductivity," Phys. Rev. B 99, 165112 (2019).

[55] Fengcheng Wu, "Topological chiral superconductivity with spontaneous vortices and supercurrent in twisted bilayer graphene,” Phys. Rev. B 99, 195114 (2019).

[56] Fengcheng Wu and Sankar Das Sarma, "Identification of superconducting pairing symmetry in twisted bilayer graphene using in-plane magnetic field and strain,” Phys. Rev. B 99, 220507 (2019).

[57] Fengcheng Wu and Sankar Das Sarma, "Ferromagnetism and superconductivity in twisted double bilayer graphene," Phys. Rev. B 101, 155149 (2020). 
[58] Ya-Hui Zhang, Dan Mao, Yuan Cao, Pablo Jarillo-Herrero, and T. Senthil, "Nearly flat chern bands in moiré superlattices," Phys. Rev. B 99, 075127 (2019).

[59] Bheema Lingam Chittari, Guorui Chen, Yuanbo Zhang, Feng Wang, and Jeil Jung, "Gate-tunable topological flat bands in trilayer graphene boron-nitride moiré superlattices," Phys. Rev. Lett. 122, 016401 (2019).

[60] Jong Yeon Lee, Eslam Khalaf, Shang Liu, Xiaomeng Liu, Zeyu Hao, Philip Kim, and Ashvin Vishwanath, "Theory of correlated insulating behaviour and spin-triplet superconductivity in twisted double bilayer graphene," Nature communications $\mathbf{1 0}$, 1-10 (2019).

[61] Xiao-Chuan Wu, Anna Keselman, Chao-Ming Jian, Kelly Ann Pawlak, and Cenke Xu, "Ferromagnetism and spin-valley liquid states in moiré correlated insulators," Phys. Rev. B 100, 024421 (2019).

[62] Tamaghna Hazra, Nishchhal Verma, and Mohit Randeria, "Bounds on the superconducting transition temperature: Applications to twisted bilayer graphene and cold atoms," Phys. Rev. X 9, 031049 (2019).

[63] Fang Xie, Zhida Song, Biao Lian, and B. Andrei Bernevig, "Topology-bounded superfluid weight in twisted bilayer graphene," Phys. Rev. Lett. 124, 167002 (2020).

[64] A. Julku, T. J. Peltonen, L. Liang, T. T. Heikkilä, and P. Törmä, "Superfluid weight and berezinskii-kosterlitz-thouless transition temperature of twisted bilayer graphene," Phys. Rev. B 101, 060505 (2020).

[65] Xiang Hu, Timo Hyart, Dmitry I. Pikulin, and Enrico Rossi, "Geometric and conventional contribution to the superfluid weight in twisted bilayer graphene," Phys. Rev. Lett. 123, 237002 (2019).

[66] Jianpeng Liu and Xi Dai, "Anomalous hall effect, magnetooptical properties, and nonlinear optical properties of twisted graphene systems," npj Computational Materials 6, 57 (2020).

[67] Yu Saito, Jingyuan Ge, Kenji Watanabe, Takashi Taniguchi, and Andrea F. Young, "Independent superconductors and correlated insulators in twisted bilayer graphene," Nature Physics 16, 926-930 (2020).

[68] Ya-Ning Ren, Chen Lu, Yu Zhang, Si-Yu Li, Yi-Wen Liu, Chao Yan, Zi-Han Guo, Cheng-Cheng Liu, Fan Yang, and Lin He, "Spectroscopic evidence for a spin- and valley-polarized metallic state in a nonmagic-angle twisted bilayer graphene," ACS Nano (2020), 10.1021/acsnano.0c04631.

[69] Si-Yu Li, Yu Zhang, Ya-Ning Ren, Jianpeng Liu, Xi Dai, and Lin He, "Experimental evidence for orbital magnetic moments generated by moiré-scale current loops in twisted bilayer graphene," Phys. Rev. B 102, 121406 (2020).

[70] Ya-Hui Zhang, Dan Mao, and T. Senthil, "Twisted bilayer graphene aligned with hexagonal boron nitride: Anomalous hall effect and a lattice model," Phys. Rev. Research 1, 033126 (2019).

[71] Nick Bultinck, Shubhayu Chatterjee, and Michael P. Zaletel,
"Mechanism for anomalous hall ferromagnetism in twisted bilayer graphene," Phys. Rev. Lett. 124, 166601 (2020).

[72] Jianpeng Liu, Zhen Ma, Jinhua Gao, and Xi Dai, "Quantum valley hall effect, orbital magnetism, and anomalous hall effect in twisted multilayer graphene systems," Phys. Rev. X 9, 031021 (2019).

[73] Fengcheng Wu and Sankar Das Sarma, "Collective excitations of quantum anomalous hall ferromagnets in twisted bilayer graphene,” Phys. Rev. Lett. 124, 046403 (2020).

[74] Manato Fujimoto, Henri Koschke, and Mikito Koshino, “Topological charge pumping by a sliding moiré pattern," Phys. Rev. B 101, 041112 (2020).

[75] Ying Su and Shi-Zeng Lin, "Topological sliding moiré heterostructure," Phys. Rev. B 101, 041113 (2020).

[76] Yinhan Zhang, Yang Gao, and Di Xiao, "Topological charge pumping in twisted bilayer graphene," Phys. Rev. B 101, 041410 (2020).

[77] Jihang Zhu, Jung-Jung Su, and Allan H MacDonald, "The curious magnetic properties of orbital chern insulators," (2020), arXiv:2001.05084 [cond-mat.mes-hall].

[78] Chunli Huang, Nemin Wei, and Allan MacDoanld, "Current Driven Magnetization Reversal in Orbital Chern Insulators," arXiv:2007.05990 [cond-mat] (2020), arXiv: 2007.05990.

[79] See Supplemental Material for (a) the derivation of Lagrangian multiplier for current, (b) the explicit calculation of the toy model, (c) the dependence on the gate distance, (d) the switching of valley polarization at zero temperature, and (e) estimate the edge current contribution in overcoming the energy barrier, which includes Refs. [85, 86].

[80] N. D. Mermin and H. Wagner, "Absence of ferromagnetism or antiferromagnetism in one- or two-dimensional isotropic heisenberg models,” Phys. Rev. Lett. 17, 1133-1136 (1966).

[81] Ming-Che Chang and Qian Niu, "Berry phase, hyperorbits, and the hofstadter spectrum: Semiclassical dynamics in magnetic bloch bands," Phys. Rev. B 53, 7010-7023 (1996).

[82] Di Xiao, Junren Shi, and Qian Niu, "Berry phase correction to electron density of states in solids," Phys. Rev. Lett. 95, 137204 (2005).

[83] Davide Ceresoli, T. Thonhauser, David Vanderbilt, and R. Resta, "Orbital magnetization in crystalline solids: Multiband insulators, chern insulators, and metals," Phys. Rev. B 74, 024408 (2006).

[84] Di Xiao, Ming-Che Chang, and Qian Niu, "Berry phase effects on electronic properties," Rev. Mod. Phys. 82, 1959-2007 (2010).

[85] G Grosso and G Pastori Parravicini, Solid State Physics (Academic Press, London, 2000).

[86] F. D. M. Haldane, "Model for a quantum hall effect without landau levels: Condensed-matter realization of the "parity anomaly”," Phys. Rev. Lett. 61, 2015-2018 (1988) 


\section{Supplemental Material: Switching of valley polarization and topology in twisted bilayer graphene by electric currents}

\section{Lagrangian multiplier for current}

The simplest way to derive the Lagrangian multiplier term in Eq. (1) in the main text is considering a torus geometry with a threading magnetic flux. The torus geometry preserves the translational invariance in both directions and the momentum is a good quantum number. The magnetic flux generates a current. The flux can be introduced into the Hamiltonian through the standard Peierls substitution $\boldsymbol{k} \rightarrow \boldsymbol{k}+\boldsymbol{e} \boldsymbol{A} / \hbar$. The single particle Hamiltonian becomes

$$
\mathcal{H}_{0}=\varepsilon(\boldsymbol{k}+e \boldsymbol{A} / \hbar) c_{k}^{\dagger} c_{k} \approx\left[\varepsilon(\boldsymbol{k})+\frac{e \boldsymbol{A}}{\hbar} \frac{\partial \varepsilon_{\boldsymbol{k}}}{\partial \boldsymbol{k}}\right] c_{k}^{\dagger} c_{k},
$$

which has the same form as the Lagrangian multiplier term in the main text. The corresponding current is given by $\boldsymbol{J}=-\partial \mathcal{H} / \partial \boldsymbol{A}$. In the absence of dissipation, a stationary current can be induced by a static flux. When dissipation is present, a time dependent flux is required to generated a stationary current. The current dissipates over a time scale associated with the quasiparticle lifetime $t_{0}$. Heuristically, we can take $\boldsymbol{A}=\boldsymbol{E}_{\boldsymbol{e}} t_{0}$, where $\boldsymbol{E}_{e}$ is the electric field. Therefore, the Lagrangian multiplier in Eq. (1) is $\lambda=-e t_{0} E_{e} / \hbar$.

The meaning of the Lagrangian multiplier can also be understood intuitively using the the Boltzmann transport equation

$$
\frac{\partial f}{\partial t}+\frac{\partial f}{\partial \boldsymbol{r}} \cdot \boldsymbol{v}+\frac{\partial f}{\hbar \partial \boldsymbol{k}} \cdot(-e) \boldsymbol{E}_{e}=-\frac{f-f_{0}}{t_{0}}
$$

under the relaxation time approximation [S1]. Here $f_{0}$ is the Fermi-Dirac function and $f$ is the distribution function in the presence of the bias voltage. For an uniform electric field $\boldsymbol{E}_{e}$, the distribution function in the stationary state becomes

$f\left(\varepsilon_{\boldsymbol{k}}\right)=f_{0}\left(\varepsilon_{\boldsymbol{k}}\right)+\frac{e t_{0}}{\hbar} \frac{\partial f_{0}\left(\varepsilon_{\boldsymbol{k}}\right)}{\partial \varepsilon_{\boldsymbol{k}}} \frac{\partial \varepsilon_{\boldsymbol{k}}}{\partial \boldsymbol{k}} \cdot \boldsymbol{E}_{e} \approx f_{0}\left(\varepsilon_{\boldsymbol{k}}+\frac{e t_{0}}{\hbar} \frac{\partial \varepsilon_{\boldsymbol{k}}}{\partial \boldsymbol{k}} \cdot \boldsymbol{E}_{e}\right)$.

Then we can identify the Lagrangian multiplier as $\lambda=$ $-e t_{0} E_{e} / \hbar$, that is consistent with the derivation above.

\section{Explicit calculation of the toy model}

Here we provide explicit calculation of the toy model using the dispersion relation $\varepsilon_{k,-}=\cos (2 k+\pi)-\mu$ for $-\pi \leq k \leq$ $-\pi / 2$ and $\varepsilon_{k,-}=\cos \left[\frac{2}{3}(k+\pi / 2)\right]-\mu$ for $-\pi / 2 \leq k \leq \pi$, and $\varepsilon_{k,+}=\varepsilon_{-k,-}$. The results for $\Delta_{ \pm}$and current $J$ as a function of $\lambda$ are displayed in Fig. S1. The switching of VP occurs only for a positive $\lambda$ because we start with the initial state shown in Fig. 1(a). For $V=3$ and $\mu=1.5$, the switching of VP (featured by the crossing of $\Delta_{ \pm}$) happens simultaneously with the insulator-to-metal transition, as shown in Figs. S1(a) and $\mathrm{S} 1$ (b). When the gap is increased by choosing $V=5$, the
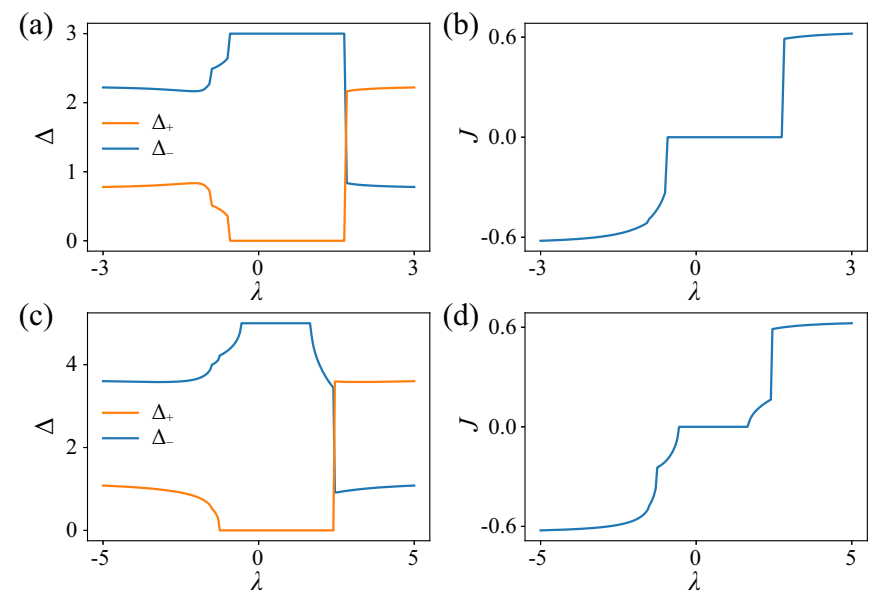

FIG. S1. (a) and (c) $\Delta_{ \pm}$as a function of $\lambda$ for $V=3$ and 5. The corresponding current $J$ are shown in (b) and (d), respectively.

system first becomes a metal, and then switches the VP upon further increasing $\lambda$, see Figs. S1(c) and S1(d).

The free energy of the system is

$$
\mathcal{F}=-k_{B} T \sum_{k, \tau} \ln \left[1+\exp \left(-E_{k, \tau} / k_{B} T\right)\right]-\frac{\Delta_{+} \Delta_{-}}{V}
$$

When the VP order parameter $\delta=\left(\Delta_{+}-\Delta_{-}\right) / 2$ is small, we can expand $\mathcal{F}$ in terms of $\delta$ as shown in Eq. (2). The coefficients are

$$
\mathcal{F}_{0}=-k_{B} T \sum_{k, \tau} \ln \left[1+\exp \left[-\left(\varepsilon_{k, \tau}-\lambda \partial_{k} \varepsilon_{k, \tau}+\rho\right) / k_{B} T\right]\right]-\frac{\rho^{2}}{V},
$$

$$
\alpha_{1}=-\sum_{k, \tau} \frac{\tau}{1+\exp (g)}
$$

$$
\alpha_{2}=-\frac{1}{2} \sum_{k, \tau} \frac{1}{2 k_{B} T+2 k_{B} T \cosh (g)}+\frac{1}{V}
$$

$$
\begin{gathered}
\alpha_{3}=-\frac{1}{6} \sum_{k, \tau} \frac{2 \tau \operatorname{csch}^{3}(g) \sinh ^{4}(g / 2)}{k_{B}^{2} T^{2}}, \\
\alpha_{4}=-\frac{1}{24} \sum_{k, \tau} \frac{[-2+\cosh (g)] \operatorname{sech}^{4}(g / 2)}{8 k_{B}^{3} T^{3}},
\end{gathered}
$$

with $\rho=\left(\Delta_{+}+\Delta_{-}\right) / 2$ and $g=\left(\varepsilon_{k, \tau}-\lambda \partial_{k} \varepsilon_{k, \tau}+\rho\right) / k_{B} T$. In the absence of current $\lambda=0, \alpha_{1}=\alpha_{3}=0$ as required by the $Z_{2}$ symmetry associated with the VP. One can show explicitly that $\alpha_{1,3}(\lambda)=-\alpha_{1,3}(-\lambda)$ and $\alpha_{2,4}(\lambda)=\alpha_{2,4}(-\lambda)$. 


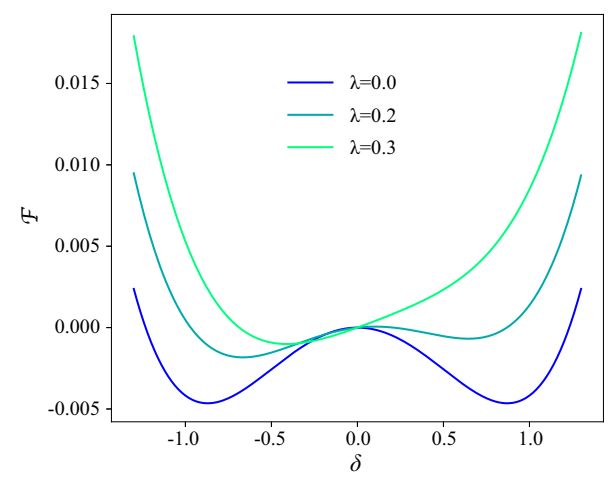

FIG. S2. Free energy as a function of $\delta$ for different $\lambda$ and $V=3$.

The free energy as a function of $\delta$ for different $\lambda$ and with $V=3$ and $\mu=1.5$ is shown in Fig. S2. The presence of a nonzero $\lambda$ lifts the degeneracy between the two valleypolarized states. As a consequence, one valley-polarized state becomes metastable, while the other becomes the global ground state. Upon further increasing or decreasing $\lambda$, the metastable state becomes unstable and the switching of VP happens.

\section{Dependence on the gate distance}

The gate distance $d$ controls the screening of the Coulomb interaction. As $d$ decreases, the screened Coulomb potential becomes weaker. When the Coulomb interaction is not overscreened, the valley-spin-polarized state in Fig. 3 remains the ground state and the QAHE persists. The energy gap of the valley-spin-polarized QAH state increases with $d$, as shown in Fig. S3. Since the robustness of the QAH state depends on the gap, the critical temperature for the QAHE and the critical current for the switching of valley polarization both increase with $d$.

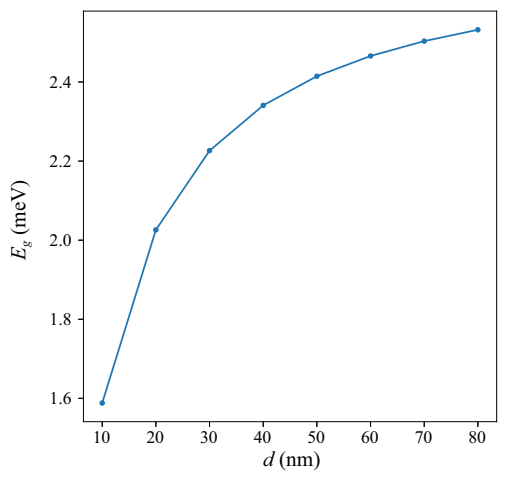

FIG. S3. Energy gap of the valley-spin-polarized QAH state as a function of the gate distance $d$.
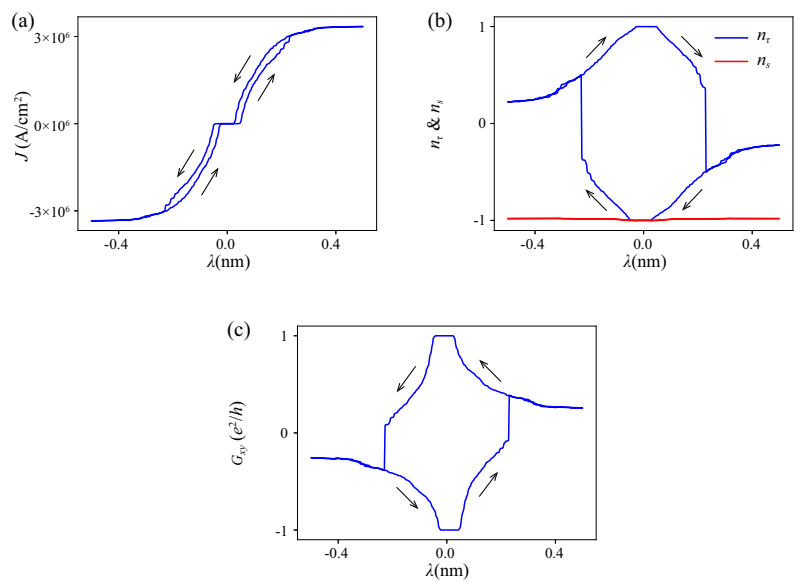

FIG. S4. (a)-(c) Current density $J$, order parameters $n_{\tau}$ and $n_{s}$, and Hall conductance $G_{x y}$ as a function of $\lambda$ at $T=0 K$. The arrows indicate the direction of evolution of the hysteresis loops.

\section{Switching of valley polarization at zero temperature}

In Fig. S4, we show the current density $J$, order parameters $n_{\tau}$ and $n_{s}$, and Hall conductance $G_{x y}$ as a function of $\lambda$ at $T=$ $0 \mathrm{~K}$. The results exhibit that a critical voltage is required to induce a current before the switching of the Hall conductance. Here the voltage $V_{e}=E_{e} L \propto \lambda$, where $L$ is the length of the system. The critical voltage is determined by the insulating gap. Above the critical voltage, electrons gain enough energy to overcome the insulating gap to conduct current.

\section{Estimate the edge current contribution in overcoming the energy barrier}

Here we estimate the edge current contribution in overcoming the energy barrier between different ground states with opposite VP. Because of the large unit cell size at a small twist angle, it is hard to access the edge states of the TBG. Instead, we use the edge states of the Haldane model to mimic that of TBG. Explicitly, we consider the Haldane model described by the Hamiltonian [S2]

$$
\begin{aligned}
\mathcal{H}_{H} & =2 t_{2} \cos \phi \sum_{i=1}^{3} \cos \left(\boldsymbol{k} \cdot \boldsymbol{b}_{i}\right) \sigma_{0} \\
& +t_{1} \sum_{i=1}^{3}\left[\cos \left(\boldsymbol{k} \cdot \boldsymbol{a}_{i}\right) \sigma_{x}+\sin \left(\boldsymbol{k} \cdot \boldsymbol{a}_{i}\right) \sigma_{y}\right] \\
& +\left[M-2 t_{2} \sin \phi \sum_{i=1}^{3} \sin \left(\boldsymbol{k} \cdot \boldsymbol{b}_{i}\right)\right] \sigma_{z},
\end{aligned}
$$

on a honeycomb lattice whose lattice constant is set to unity. Here $t_{n}$ is the hopping energy between the $n$th nearest neighboring (NN) lattice sites, $\sigma_{0}$ is the $2 \times 2$ identity matrix, and $\sigma_{x, y, z}$ are the Pauli matrices acting on the sublattice degree of 

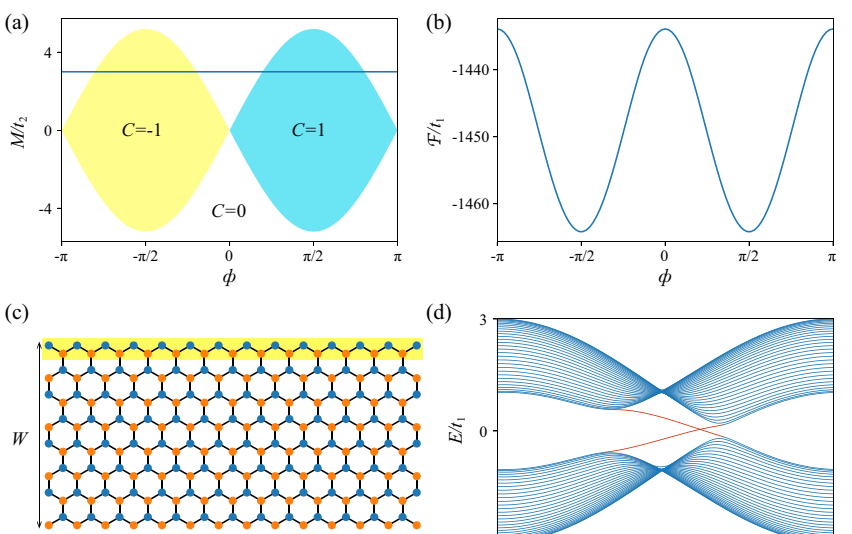

FIG. S5. (a) Phase diagram of the Haldane model. The Chern numbers of different phases are shown. (b) Free energy of the Haldane model along the straight line in (a) at half filling and zero temperature. (c) A strip of honeycomb lattice with width $W$ and zigzag edges. (d) Energy spectrum of the Haldane model on the honeycomb lattice strip.

freedom. $\boldsymbol{a}_{1,2,3}$ are the three vectors connecting NN lattice sites, and $\boldsymbol{b}_{1,2,3}$ are primitive lattice vectors related by 3 -fold rotation. $M$ is the sublattice potential breaking the inversion symmetry, and $\phi$ is the phase factor associated with the second NN hopping. The topological phase of the model is determined by $M$ and $\phi$, as shown by the phase diagram in Fig. S5(a). By choosing $t_{2}=0.07 t_{1}$ and $M=3 t_{2}$, we show the free energy as a function of $\phi$ of the Haldane model at half filling and zero temperature in Fig. S5(b). Apparently, there are two degenerate energy minimum with opposite Chern number $C= \pm 1$ at $\phi= \pm \pi / 2$, that is similar to the TBG. Therefore, by taking $\phi$ as an internal order parameter (playing the same role of $\delta$ ), we can use the Haldane model to mimic the free energy profile and topology of the TBG.

To study the edge current contribution, we consider a stripe of honeycomb lattice with zigzag edges along the $x$ direction, as shown in Fig. S5(c). The edge asymmetry can be introduced by adding an onsite potential $V$ to the upper edge, which is highlighted in Fig. S5(c). For $V=2 t_{2}$, the energy spectrum of the strip with width $W=25 \sqrt{3}$ (that contains 50 unit cells in the cross section) is shown in Fig. S5(d). Here the edge states traversing the the bulk energy gap are marked by red color. In the presence of a current along the strip, the system is described by $\mathcal{H}_{H}-\sum_{n} \lambda \partial_{k_{x}} \varepsilon_{k, n}$ where $\varepsilon_{k, n}$ represents the bare dispersion of the strip [see Fig. S5(d)] and $n$ is the band index. The current lifts the degeneracy of the two energy minima at $\phi= \pm \pi / 2$, as shown in Fig. S6(a). The energy barrier between the two minima vanishes at a critical $\lambda$, i.e. $\lambda_{c} \approx 1.25$ in this case, that corresponds to the switching of VP and topology in TBG. To show the edge current contribution in overcoming the energy barrier, we study the electron occu-

pation in the conductance and valence bands at $\lambda=\lambda_{c}$. As a reference, we show the electron occupation in the absence of current for $\lambda=0$ in Fig. S6(b), where the conductance (valence) bands are fully empty (occupied) at zero temperature. The two branches of counter-propagating edge states are partially filled. In the presence of the critical current for $\lambda=\lambda_{c}$, the electron occupation is shown in Fig. S6(c). Apparently, the branch of edge states propagating along the current direction is fully filled, while the other branch is empty. Moreover, there are electrons pumped from the valence bands to conductance bands as in the TBG. Namely, the edge current alone is not enough to overcome the energy barrier in this case. The pumped electrons occupy the the bottom of conduction bands where the density of states is high. Therefore, the bulk current plays a dominant role in overcoming the energy barrier for a large system. The edge current can be important if the sample size is small since the energy barrier grows linearly in the sample volume. However, in experiments [6,7], the device is in micron size, that contains hundreds of moire unit cells (considering the lattice constant of the small-angle TBG is of the order of $10 \mathrm{~nm}$ ) in the cross section. This fact motivates us to study the switching of VP and topology in TBG by electric currents in the bulk.
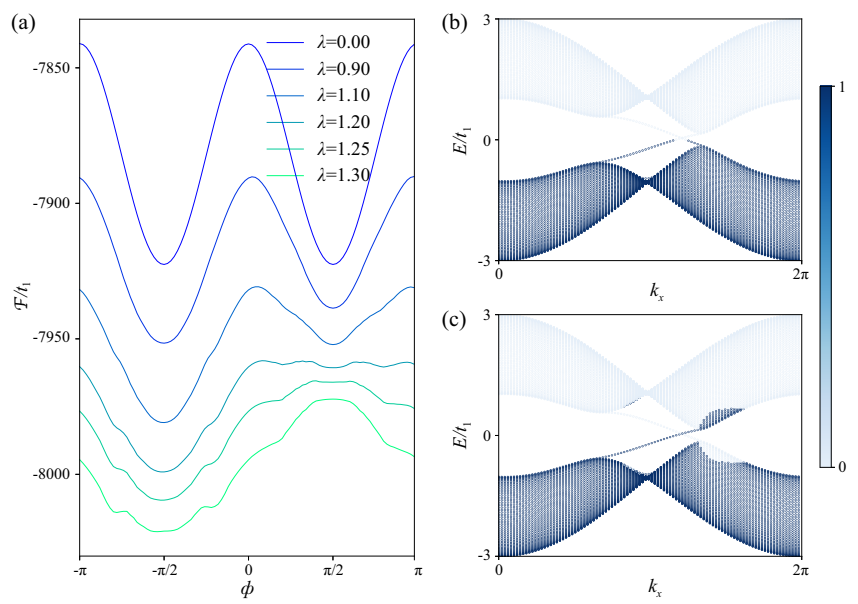

FIG. S6. (a) Free energy of the Haldane model as a function of $\phi$ for different $\lambda$. (b) and (c) Electron occupation for $\lambda=0$ and for $\lambda=1.25$, respectively.

[S1] G. Grosso and G. P. Parravicini, Solid State Physics (Academic Press, London, 2000).

[S2] F. D. M. Haldane, Model for a Quantum Hall Effect without Landau Levels: Condensed-Matter Realization of the "Parity Anomaly”, Phys. Rev. Lett. 61, 2015 (1988). 